\title{
Option pricing and hedging for optimized Lévy driven stochastic volatility models
}

\author{
Xiao-li GONG* Xin-tian ZHUANG \\ School of Business Administration, Northeastern University, Shenyang, 110169, China
}

\begin{abstract}
This paper pays attention to Ornstein-Uhlenbeck (OU) based stochastic volatility models with marginal law given by Classical Tempered Stable (CTS) distribution and Normal Inverse Gaussian (NIG) distribution, which are subclasses of infinite activity Lévy processes and are compared to finite activity Barndorff-Nielsen and Shephard (BNS) model. They are applied to option pricing and hedging in capturing leptokurtic features in asset returns and clustering effect in volatility that are consistently observed phenomena in underlying asset dynamics. The analytical formula of option pricing can be obtained through use of characteristic functions and Fast Fourier Transform (FFT) technique. Additionally, we introduce two hybrid optimization techniques such as hybrid Particle Swarm optimization (PSO) algorithm and hybrid Differential Evolution (DE) algorithm into parameters calibration schemes to improve the calibration quality for newly constructed models. Finally, we conduct experiments on Chinese emerging option markets to examine the performance of proposed models exploiting hybrid optimization techniques.
\end{abstract}

Keywords: OU process; infinite activity Lévy jumps; hybrid PSO and DE optimization; option pricing and hedging

\section{Introduction}

The simplest assumption of constant drift and volatility coefficient of underlying asset returns dynamic that follows geometric Brownian motions contradicts with phenomena observed in financial markets. During the past decades, abundant experiments have been performed on option pricing in stochastic volatility and jumps models. Among the most widely used, the striking Lévy process and OU process based stochastic volatility models can account for the important distributional properties of returns and implied volatility smile. We pay attention to stochastic volatility models with jumps cases where the Lévy jumps are driven by infinite activity processes and finite activity process, respectively. The advantage of our constructed model over the prevailing is the reflection of infinite jump behaviors in underlying assets and clustering effect in time change volatility. In particular, due to the availability of analytical characteristic functions and suitable computational tractability of the two processes, they can thus be directly utilized for parameters estimation.

Some well-known stylized facts about asset returns are well illustrated which cover asymmetry, time-varying variance, leptokurtosis and heavy tails. Moreover, there is affluent evidence that volatility exhibits clustering and heteroskedasticity effect, leading to stochastic jumps in stock prices [1]. It has been demonstrated that stochastic volatility and jumps are inherent components of stock price dynamics [2, 3]. Many alternative models are developed to explain the intrinsic characteristics of asset returns and volatility smile in option prices [4-7]. Therefore, incorporating both stochastic volatility and jumps

\footnotetext{
* Corresponding author.

E-mail address: qdgongxiaoli@126.com (X.L. Gong)
}

becomes necessary when constructing option price models. By observing the evolution of returns, it can be easily found that the returns process is accompanied by not only large jumps, but also small jumps. The Lévy process contains infinite activity processes that generate infinite numbers of jumps within any time interval or it can be finite activity processes. Just as what Carr and $\mathrm{Wu}$ [8] have addressed that infinite activity Lévy processes can capture both large jumps and small jumps in stock price dynamics. Three different distributions belonging to the Lévy processes are analyzed in this paper, namely, the CTS distribution, the NIG distribution and the compound Poisson distribution.

The stochastic volatility models naturally become reasonable tools to solve heteroskedasticity and volatility clustering effect in returns variance. It is illustrated necessary to take into account the stochasticity of the volatility in numerous literatures, such as Valenti et al. [9], Bonanno et al. [10], Spagnolo et al. [11] and Bonanno et al. [12], where a nonlinear modified Heston model with stochastic volatility was utilized for empirical research. To overcome the shortcoming of the floating smile property, stochastic volatility extension of Lévy processes based models are considered. In order to incorporate stochastic volatility into Lévy process modeling framework, Schoutens [13] used the time change approach to let the business time be a stochastic process that reflects stochastic movements in volatility instead of calendar time. The Gamma-OU (GOU) process has been applied to the construction of stochastic volatility models for pricing options and for the modeling of default intensity in credit default swaps [14]. Barndoff-Nielsen et al. [15] constructed non-negative OU type processes by replacing Browian motion with non-negative Lévy subordinators. Creal et al. [16] examined the effectiveness of Lévy-driven OU models by filtering and smoothing algorithms. 
Although abundant work has been done on jump-diffusion stochastic volatility models [17-19], they feature a counter-factual assumption that jumps rarely occur. Thereby, we particularly introduce two infinite pure jumps distributions that could be superposed to stochastic volatility models, which are Normal Inverse Gaussian processes proposed by Barndorff-Nielsen [20], Classical Tempered Stable processes presented by Rosinski [21]. Dubkov [22] introduced the Lévy flight superdiffusion to convert the infinitely divisible characteristic function of the Lévy processes which are extensively applied to finance by Kim and Rachev [23], Kuchler et al. [24] later. Tempered stable processes are the product of the Lévy measure of $\alpha$ stable processes multiply different tempered functions. Although $\alpha$ stable processes can characterize the high peak nature in financial assets, their tail distributions are too thick to capture real tail behaviors in markets. After multiplying tempered functions, they get intermediate between normal distributions and stable distributions appropriately so as to describe the fat tail behaviors of financial data. Zaevski et al. [25], Li et al. [26] and Liang et al. [27] verified the efficiency of this class models by introducing tempered stable Lévy jumps into Cox-Ingersoll-Ross stochastic clock based stochastic volatility models for SP 500 option pricing. Then we develop the GOU based stochastic volatility Lévy processes (LVGOU) models by subordinating Lévy processes to the stochastic volatility models. Noticeably, the BNS model is a special case of stochastic volatility Lévy processes that takes place the infinite jumps components into Poisson jumps.

A major difficulty in implementing traditional parameters estimation methods to GOU based Lévy processes models is the lack of analytical transition density form and the demanding computation of higher derivatives. Furthermore, model calibration to market options data formulates a nonlinear optimization problem which often suffers from local minima problems, thus leading to poor performance in analyzing the behavior of derivative markets. As pointed out in $\mathrm{Yu}$ et al. [28], it is significant to obtain an accurate parameter set that calibrates the cross-sectional data well for the proposed models so that the model can be effectively applied in finance. Taufer et al. [29], Kawai et al. [30], Zhang et al. [31] investigated the random numbers simulation algorithm of GOU based Lévy processes models processes so as to apply it in maximum likelihood estimation, whereas the more general calibration approach to this family is the intelligence optimization algorithm. Fortunately, most analytical characteristic functions of GOU based Lévy processes models models are obtainable. Yang and Lee [32] proposed multi-basin PSO for parametric Lévy models calibration in selecting initial particles set and illustrated that it outperforms local search techniques and other intelligent algorithms. Fastrich et al. [33] utilized DE algorithm on German stock for portfolio forecast. To verify the performance of global intelligent search methods, we apply hybrid PSO technique and hybrid DE technique to the developed LVGOU models to examine the performance of newly constructed models. Then simulations are carried out on both model prices and Hangseng index (HSI) option prices in Chinese Hong kong market.

The organization of the paper is as follows. Section 2 builds the OU based stochastic volatility models with Lévy jumps by subordinating the extensively used infinite activity Lévy processes to stochastic volatility process. Section 3 briefly introduces the hybrid PSO algorithm and the hybrid DE algorithm respectively. In Section 4 we present Carr-Madan's Fourier transform approach and the FFT technique. Then we exhibit the empirical researches about option pricing as well as option hedging in Section 5, finally we conclude the paper in Section 6.

\section{Lévy processes driven stochastic volatility models}

\subsection{Ornstein-Uhlenbeck process}

The random variation in volatility can be featured by instantaneous changes in time. Additional parameters are needed to allow for modifying the timely evolution of volatility. One typical example of the rate of time change used to induce stochastic volatility is the Gamma Ornstein-Uhlenbeck process. The Gamma Ornstein-Uhlenbeck process serves as a measure of time change rate in which it speeds up or slows down the time elapse rate to increase or decrease the activity intensity so that it can capture the volatility clustering and heteroskedasticity effect. Here we choose the GOU process which satisfies the stochastic differential equation under risk neutral measure

$d v_{t}=-\lambda v_{t} d t+d z_{\lambda_{t}}$,

where $z_{\lambda t}=\sum_{n=1}^{N_{t}} x_{n}, N_{t}$ denotes Poisson process with intensity $a$ and exponentially distributed jumps, and each sequence $x_{n}$ follows an exponential law with mean $1 / b$. After scaled with $\lambda$, this process of $z_{\lambda t}$ is stationary with Gamma limit distribution.

Since it is driven by a Gamma process, the typical path is not continuous with jumps decaying exponentially until the next jump. The integrated process of $v_{t}$ is defined as $V_{t}=\int_{0}^{t} v_{s} d s$, and variable $v_{t}$ features the intensity of economic activities. Randomly changed time can be seen as business time, the randomness in it generates stochastic volatility. The time changing approach is efficient because when the variance rate is high, business time flows faster as well as jumps occur frequently. The characteristic function of Gamma Ornstein-Uhlenbeck process has a closed form solution that can be rewritten as

$V_{t}=\lambda^{-1}\left(1-e^{-\lambda t}\right) \lambda_{0}+\lambda^{-1} \int_{0}^{t}\left(1-e^{-\lambda(t-s)}\right) d z_{\lambda_{s}}$.

\subsection{Infinite activity Lévy processes}


One can define a cadlag process $X(t)$ an infinite divisible Lévy process that has independent, stationary increments and stochastic continuity in filtration space $(\Omega, F, P)$. In Sato [34] the infinite divisible Lévy distribution has a triplet $(\mu, \sigma, v)$ of characteristics, which respectively represent a linear deterministic part, a Brownian motion part and a pure jump part.

Considering the complicated distributions functions representations for Lévy processes, we generally use characteristic function $\varphi(u)=E\left[e^{i u X(t)}\right]$, or characteristic exponent $\psi(u)=(1 / t) \log \varphi(u)$ for calculation facility. According to Lévy-Khintchine formula, the characteristic function of infinite divisible distribution $X(t)$ satisfies the following $E[\exp (i u X)]$

$=\exp \left(-i u \mu+\frac{\sigma^{2} u^{2}}{2}-\int_{-\infty}^{+\infty}\left(e^{i u x}-1-i u x_{|x| \leq 1}\right) v d x\right)$

where real number $\mu$ refers to the drift that determines the location, $v$ denotes the Lévy measure which indicates the arrival rate of jumps.

In order to make sure that $e^{-r t} S_{t}$ is a martingale, additional restrictions should be exerted on the triplet, that is, $\int_{|x| \geq 1} e^{x} v(d x)<\infty$ and $E^{Q}\left[e^{X_{t}}\right]=e^{t \psi_{X}(-i)}=1$ for all $t$ in the risk neutral martingale measure $Q$. We briefly outline two widely employed infinite activity Lévy processes, that is Normal Inverse Gaussian model and Classical Tempered Stable model below.

The Normal Inverse Gaussian distribution with parameter $\alpha>0,-\alpha<\beta<\alpha, \delta>0$, NIG $(\alpha, \beta, \delta)$ has a characteristic function given by

$$
\begin{aligned}
& \varphi_{N I G}(u ; \alpha, \beta, \delta)= \\
& \exp \left(-\delta\left(\sqrt{\alpha^{2}-(\beta+i u)^{2}}-\sqrt{\alpha^{2}-\beta^{2}}\right)\right)
\end{aligned}
$$

It is clearly shown that this is an infinitely divisible characteristic function. The parameter $\beta$ in NIG model governs the direction premium, and it is positively related to the variance, skewness and kurtosis of the density and parameter $\delta$ affects the kurtosis of returns distribution. Because of the rich parameters set, it can generate a variety of distribution forms. Model parameters above directly affect the Lévy measure and probability density distribution in returns. The Lévy measure determining the jumping behavior is given by

$v(d x)=\frac{\alpha \delta}{\pi} \frac{\exp (\beta x) K_{1}(\alpha|x|)}{|x|} d x$,

where $K_{1}($.$) is the modified Bessel function of the$ second kind.

The Classical Tempered Stable model is a Lévy model with no Gaussian component. Let $\alpha \in(0,1) \cup(1,2)$ to guarantee the finite quadratic variation, $C, \lambda_{+}$and $\lambda_{-}>0, m \in \mathrm{R} . X$ is said to follow the CTS distribution if the characteristic exponent of $X$ is given by

$\psi_{C T S}\left(u ; \alpha, C, \lambda_{+}, \lambda_{-}\right)=-C \Gamma(1-\alpha)\left(\lambda_{+}^{\alpha-1}-\lambda_{-}^{\alpha-1}\right)$,

$+C \Gamma(-\alpha)\left(\left(\lambda_{+}-i u\right)^{\alpha}-\lambda_{+}^{\alpha}+\left(\lambda_{-}+i u\right)^{\alpha}-\lambda_{-}^{\alpha}\right)$

where $\Gamma$ stands for the gamma function and $\alpha \neq 0,1$.

The scale parameter $C$ in CTS model reflects the jumps frequency and is related to the kurtosis of the density function, where smaller $C$ leads to higher peak.

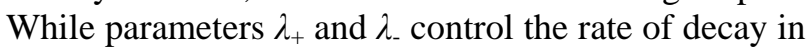
positive and negative tails respectively. If $\lambda_{+}$is not equal to $\lambda_{-}$, then it is asymmetric in jumps structures. The stable index $\alpha$ determines the price evolution between jumps.

And the Lévy measure of CTS distribution can be written as

$v(z)=q(z) v_{S_{\alpha}}(z)=\frac{C e^{-\lambda_{+} z}}{z^{1+\alpha}} 1_{z>0}+\frac{C e^{-\lambda_{-}|z|}}{|z|^{1+\alpha}} 1_{z<0}$,

where $q(z)$ refers to the tempering function of the CTS law, $v_{s \alpha}$ is the Lévy measure of $\alpha$ stable law.

\subsection{The stochastic volatility Lévy processes}

The characteristic function of the stochastic volatility Lévy models can be composed from the characteristic exponent of Lévy component and characteristic function of the rate of time change. The economic time passed in calendar time is made stochastic by the stochastic clock built in through integral of time. The characteristic function of Gamma Ornstein-Uhlenbeck process given in (1) is expressed as follows

$$
\begin{aligned}
\varphi(u)= & \exp \left[i u y_{0} \lambda^{-1}\left(1-e^{-\lambda t}\right)+\right. \\
& \left.\frac{\lambda a}{i u-\lambda b}\left(b \ln \left(\frac{b}{b-i u \lambda^{-1}\left(1-e^{-\lambda t}\right)}\right)-i u t\right)\right]
\end{aligned}
$$

The Lévy processes driven stochastic volatility models incorporates jumps and stochastic volatility simultaneously and eventually obtains three models denoted as Barndorff-Nielsen and Shephard (BNS) model, GOU based Normal Inverse Gaussian (NIGGOU) model and GOU based Classical Tempered Stable (CTSGOU) model respectively by subordinating Lévy processes to the stochastic clock. According to the derivation of Schoutens [13], characteristic functions of GOU based Lévy processes processes for returns are the following form

$$
\begin{aligned}
E[\exp (i u S(t))] & =E\left[\exp \left(V(t) \psi_{X}(u)\right)\right] \\
& =\varphi\left(-i \psi_{X}(u), t, v(0) ; a, b, \lambda\right)
\end{aligned} .
$$

We evaluate the characteristic function of integrated OU processes with Lévy components in a practical application view. The characteristic functions of risk neutral stock price processes are obtained by a mean correcting factor $\exp ((r-q) t) / E\left[\exp (S(t)) \mid v_{0}\right]$, where $r$ and $q$ represents constant continuously compound interest rate and dividend yield respectively.

One stochastic volatility Lévy process model that driven by compound Poisson jumps is the finite activity jump diffusion stochastic volatility model, namely BNS model, which contains simultaneous upward jumps in volatility and downside jumps in underlying prices, with the risk-neutral dynamics given by

$$
\begin{aligned}
d\left(\log \left(S_{t}\right)\right)= & \left(r-\lambda k(-\rho)-\xi_{t}^{2} / 2\right) d t \\
& +\xi_{t} d W_{t}+\rho d z_{\lambda t}
\end{aligned}
$$


where the squared volatility process follows $d \sigma_{t}^{2}=-\lambda \sigma_{t}^{2} d t+d z_{\lambda t}$, and $\mathrm{Z}_{\lambda t}$ denotes the same meaning as in (1). There are totally five parameters containing $\xi, \rho, \lambda, a, b$.

Other LVGOU models such as NIGGOU and CTSGOU models describe infinite jumps behaviors. The characteristic function for the NIGGOU process is given by

$$
\begin{aligned}
\varphi_{\text {NIGGOU }}= & \exp \left(i u\left(\log \left(S_{0}+(r-q) t\right)\right)\right. \\
& \times \frac{\varphi\left(-i \psi_{N I G}(u ; 1, \alpha, \beta), t, \delta ; a, b, \lambda\right)}{\varphi\left(-i \psi_{N I G}(-i ; 1, \alpha, \beta), t, \delta ; a, b, \lambda\right)^{i u}},
\end{aligned}
$$

where there are six parameters consisting of $\alpha, \beta, \delta, a$, $b, \lambda$ in model.

The characteristic function for the CTSGOU process is expressed as

$$
\begin{aligned}
\varphi_{\text {CTSGOU }}= & \exp \left(i u\left(\log \left(S_{0}+(r-q) t\right)\right) \times\right. \\
& \frac{\varphi\left(-i \psi_{C T S}\left(u ; 1, \lambda_{-}, \lambda_{+}, \alpha, C\right), t, C ; a, b, \lambda\right)}{\varphi\left(-i \psi_{C T S}\left(-i ; 1, \lambda_{-}, \lambda_{+}, \alpha, C\right), t, C ; a, b, \lambda\right)^{i u}}
\end{aligned}
$$

which is a nine parameters process with $C, \lambda_{+}, \lambda_{-}, \alpha, a$, $b, \lambda$.

Then, we obtain the models of stock price dynamics capturing not only jumps but also stochastic volatility. The stochastic volatility extensions of Lévy processes based models overcome the shortcoming of floating smile property in fitting to option prices. The above models also have such appealing nature that they can find analytical solutions for option prices. Therefore it is convenient for parameters estimation in reasonable amount of time. The analytical solutions can be obtained utilizing characteristic functions and taking FFT technique.

\section{Calibration optimization algorithm}

It has been illustrated that poor local solutions of calibration generate undesirable matching results and the necessity to introduce global intelligent search methods to enhance the quality of calibration procedure. The Particle Swarm optimization algorithm and Differential Evolution optimization algorithm are two popular intelligence global search methods. They are extremely effective at identifying areas where potential optima are located, but the algorithms converge relatively slowly after finding such areas. This necessitates the use of hybrid algorithms combining global and local schemes. Here, we use intelligence global search methods to identify the general location of the global optimum, and then we use local search algorithm to pinpoint their exact location, specifically, using PSO in combination with interior point algorithm, and using DE in combination with Lavenberg Marquard algorithm.

\subsection{Particle Swarm optimization algorithm}

The Particle Swarm optimization algorithm is a population based stochastic optimization approach that is extensively studied by Panda and Padhy [35].
Unlike the genetic algorithm, the Particle Swarm optimization algorithm, which is inspired by the social behaviors of bird flocking or fish schooling, has no evolution operators such as crossover and mutation. In PSO, the potential solutions, which are called particles, fly through the problem space by following the current optimum particle. The general behavior of PSO is that particles cooperatively search for the optimal; with every particle has its own velocity and flying towards the global optimum after remembering the past direction. In other words, every particle is replaced by the global best particle in its neighborhood space, and adjusts its velocity and direction. The algorithm can be expressed as

$$
\begin{aligned}
& v_{i}^{k+1}=\varepsilon^{k} v_{i}^{k}+\alpha_{1}\left[\omega_{1, i}\left(P_{i}-x_{i}^{k}\right)\right]+\alpha_{2}\left[\omega_{2, i}\left(P_{i}-x_{i}^{k}\right)\right], \\
& x_{i}^{k+1}=x_{i}^{k}+v_{i}^{k+1}
\end{aligned}
$$

where variables $x$ and $k$ denote the current location and velocity of the $i$ th particle in the $k$ th generation. The swarm is composed of $N$ particles with $P_{i}$ standing for the personal best particle and $G$ standing for the global best. $\omega_{1, i}, \omega_{2, i} \in[0,1]$, and $\varepsilon^{k}$ represents particle inertia.

The new particle velocity $v_{i}^{k+1}$ is made up of three components. The momentum term $\varepsilon^{k} v_{i}^{k}$ plays the role of keeping particles moving along present trajectory, the attraction term $\alpha_{1}\left[\omega_{1, i}\left(P_{i}-x_{i}^{k}\right)\right]$ makes particles fly towards personal best location, the attraction component $\alpha_{2}\left[\omega_{2, i}\left(P_{i}-x_{i}^{k}\right)\right]$ guides the swarm towards the global best $G$.

\subsection{Differential Evolution optimization algorithm}

The recently emerging evolution algorithms are particularly suitable to solve optimization problems encountered in the calibration of financial models. The Differential Evolution algorithm avoids becoming trapped in flat regions or local optima of the objective function. A comprehensive description of Differential Evolution algorithm is provided by Price et al. [36]. Application of this algorithm in the portfolio optimization context is in Krink and Paterlini [37]. The basic idea is to evolve a population of solutions by means of recombination, crossover, and survival of the fittest. It can be formulated as

$P_{., i}^{v}=P_{., r 1}^{0}+F \times\left(P_{., r 2}^{0}-P_{., r 3}^{0}\right)$.

In every generation, this technique creates a candidate solution for already existing solution $P_{., i}^{k}$.The new candidate is developed by primarily adding the difference, weighted as a parameter $F$ between the other two solutions to the third one. Then the crossover step is taken place with probability $C R$ between this latter generated candidate $P_{., i}^{v}$ and the already existed solution $P_{., i}^{k}$. If the latter candidate $P_{., i}^{u}$ is better than $P_{., i}^{k}$, it will replace it; if not, the old solution is kept. 


\section{FFT for Option pricing methodology}

There are three methods for pricing vanilla options with known characteristic functions of the assets returns including direct integration, Fast Fourier Transform and fractional Fast Fourier Transform. The direct integration method is computationally demanding that involves recalculation of functions, whereas the fractional FFT approach does not overcome the crucial limitation of the FFT method because of the equidistantly chosen grid points. The FFT method satisfies calculating option prices for the whole range of strikes and maturities simultaneously. So, we continue to use FFT technique.

Let $k=\ln K$ and $T$ be the maturing time of a European call option with terminal asset price $S(T)$, where $S(T)$ is governed by the GOU based stochastic volatility Lévy processes processes dynamics, and $\gamma$ be a positive constant assuring the existence of the asset price's $\gamma$ th moment. We use the transformation of the vanilla pricing formula suggested by Carr and Madan [38] that allowed using Fast Fourier Transform technique and can be used conveniently once characteristic functions of the risk neutral asset price process is available. Since the integrand in direct integration is not square integrable, the Fourier transform of the dampened call option price is needed and the formula of option pricing can be expressed as

$c(k, T)=\frac{\mathrm{e}^{-\gamma k}}{\pi} \int_{0}^{+\infty} \mathrm{e}^{-i u k} \vartheta(u) d u$,

where $\gamma$ is a dampening parameter and $\vartheta(u)=\frac{\mathrm{e}^{-r T} \phi(u-(\gamma+1) i)}{\gamma^{2}+\gamma-u^{2}+i(2 \gamma+1) u}$.

A sufficient condition of $c(k, T)$ to be integrable for both sides is given by $\vartheta(0)$ being finite, that is,

$\vartheta(0)=\frac{\phi(-(\gamma+1) i)}{\gamma^{2}+\gamma}<\infty$.

This will produce a range of $\gamma:\left(\gamma_{+}, \gamma_{-}\right)$. Let $\gamma_{\text {Max }}=\gamma_{+}-1, \gamma_{\text {Min }}=\gamma_{-}-1$, the range $\left[\gamma_{\text {Max }}, \gamma_{\text {Min }}\right]$ contains all allowed $\gamma$. According to Lord et al. [39], one should choose $\gamma$ such that

$$
\gamma=\arg \min \left[-\gamma k+\ln \phi_{F}\left(t_{0}, T,-(\gamma+1) i\right)\right] \text {. }
$$

The fast Fourier Transform technique can be utilized to expedite the calculation speed. The FFT based methods have an advantage of simultaneously calculation for all strikes. The FFT returns $N$ values of $k$ and for a regular spacing size of $h$ where the number of grid points $N$ is a power of 2 , the value for $k_{u}$ is $k_{u}=-b+h(a-1)$, with $a$ being an positive integer and $b=N h / 2$, giving us log strike levels ranging from $-b$ to $b$. The integral in (15) is approximated employing the Trapezoid rule which yields

$$
\begin{aligned}
& c\left(k_{u}\right) \approx \frac{\mathrm{e}^{-\gamma k_{u}}}{\pi} \times \\
& \sum_{j=0}^{N} \exp (-i z v(j-1)(a-1)+i b v(j-1)) \vartheta(v(j-1)) v
\end{aligned}
$$

In the FFT application, we note that $v h=2 \pi / N$, let $u_{j}=v(j-1)$, with Simpson's rule weighting, the value of European call option is

$$
\begin{gathered}
c\left(k_{u}\right) \approx \frac{\mathrm{e}^{-\gamma k_{u}}}{\pi} \sum_{j=1}^{N} \exp \left(-i \frac{2 \pi}{N}(j-1)(a-1)+i b u_{j}\right) \\
\vartheta\left(u_{j}\right) \frac{v}{3}\left[3+(-1)^{j}-\ell_{j-1}\right]
\end{gathered}
$$

where $\ell_{n}$ is the Kronecker delta function that is unity for $n=0$ and zero otherwise.

\section{Empirical results}

\subsection{Descriptive statistics of index returns}

The Hangseng Index in Hongkong and the Shanghai and Shenzhen 300 index (SS300) in Chinese mainland, as well as the Standard \& Poor 500 Index (SP500) in America are all important representative indexes of the global capital markets. We select index prices of HSI, SP500 and SS300 indexes from Wind database during 2011 to January, 2015, 1000 observations in total. The descriptive statistics of the sample returns are displayed in Table 1. And Fig. 1 exhibits the volatility process, skewness process, kurtosis evolution and noise sequences of HSI.

Table 1

Descriptive statistics of index returns.

\begin{tabular}{ccccccc}
\hline index & mean & variance & skewness & kurtosis & heteroskedasticity & $J B$ test $(p)$ \\
\hline HSI & -0.0006 & 0.0012 & -0.0824 & 6.28 & 235.3 & $1.86 \mathrm{e}-4$ \\
SS300 & $7.6538 \mathrm{e}-5$ & 0.0134 & -0.0741 & 5.6924 & 242.9 & $1.02 \mathrm{e}-3$ \\
SP500 & $6.91 \mathrm{e}-3$ & 0.0103 & -0.1255 & 7.4237 & 240.6 & $2.02 \mathrm{e}-4$ \\
\hline
\end{tabular}

The descriptive statistics in Table 1 find both left-skewed features and leptokurtosis existed in the above three indexes. Heteroscedasticity test results show remarkable heteroscedasticity effect in both the three returns, $J B$ test easily rejects the normality hypothesis of indexes returns distributions with very small $p$ value. The values of skewness and kurtosis validate characteristics of leptokurtosis asymmetry existed in HSI index, SS300 index and SP500 index. Fig. 1 plots the timely evolution processes of volatility, skewness, kurtosis and noises of HSI index. We can directly observe outstanding volatility agglomeration and mean reversion tendency in volatility process, which reflect irregular infinite jumps in stock prices 
dynamics. Obviously, there exist many outliers in noise, indicating the existence of non-Gaussian components. The skewness process validates the left skew phenomenon of returns by consistently negative
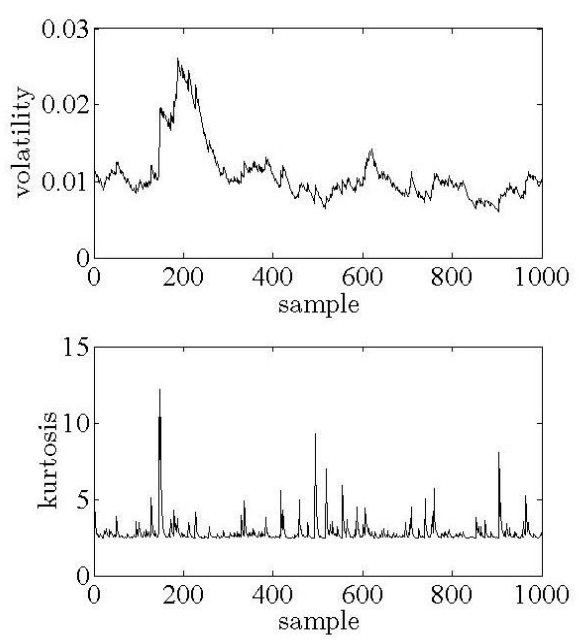

valued curves. The kurtosis values vary greater than that in normal distribution, showing excess kurtosis in returns. Table 1 and Fig. 1 collectively illustrate the stylized features of financial returns.
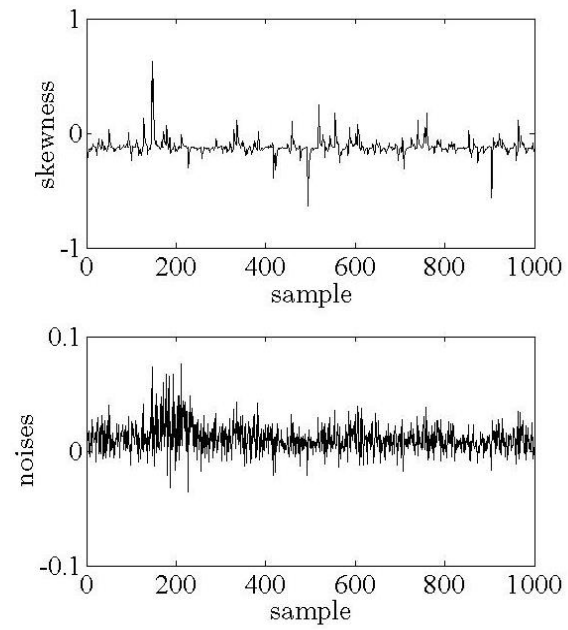

Fig. 1. The evolution of volatility, skewness, kurtosis and noise

To further compare the effectiveness of density distributions fitting, we use the time series data of HSI index and SP500 index to separately draw the density distributions of time changing Normal Inverse Gaussian model and time changing Classical Tempered Stable model compared with the historical density curve. It can be shown in Fig. 2 that the GOU based Classical Tempered Stable model is superior to infinite activity jump GOU based Normal Inverse Gaussian model at the leptokurtosis and especially the tail performance since the time changing Normal Inverse Gaussian model cannot capture the actual left skewed property and overestimate the actual peakness of returns. The Classical Tempered Stable marginal distribution is closer to real phenomenon of financial returns and more accurately reflects the distribution behavior of capital markets. Regarding the probability

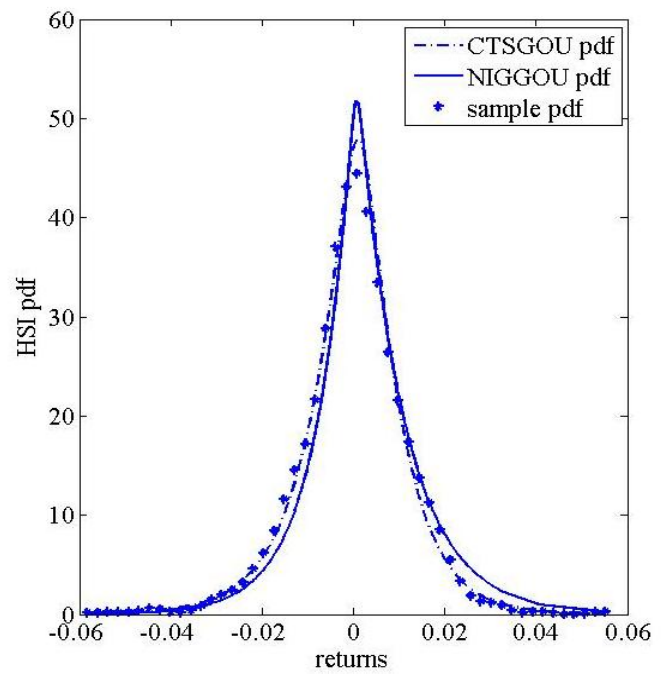

distribution of the volatility, we calculate the distribution characteristics of the volatility in HSI index returns and the simulated volatility sequences of NIGGOU model and CTSGOU model. It shows that the skewness values are 2.8292, 3.3402 and 3.1769 respectively, indicating all volatility processes are right skewed, the kurtosis values are 4.3017, 4.9526 and 4.8784 respectively, showing extreme fluctuations happen frequently in both real data and simulated volatility series, and heteroskedasticity estimations verify the existence of time changed volatility, signifying the time varying volatility processes assumption are reasonable. There is no evident differences from volatility statistics characteristics between actual volatility process and simulated volatility processes probably because the prevailing existence of clustering and persistence in them

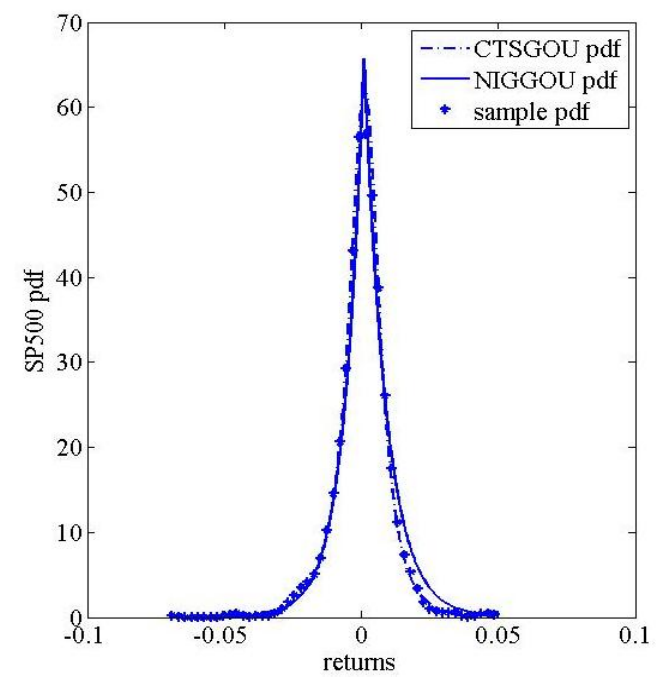

Fig. 2. Density distributions for returns in different models

For the sake of contrasting the correlation function of the SP500 returns for real data with models, we generate the random sequences numbers assuming that the stock returns follow the time changed Normal Inverse Gaussian process and time changed Classical Tempered Stable process by Monte 
Carlo simulation, respectively. Then we calculate the correlation functions of the SP500 returns and CTSGOU model which are displayed in the upper panel of Fig.3 as well as the correlation functions of SP500 absolute returns and NIGGOU model which are displayed in the lower panel of Fig.3, where he blue line represents the boundary line of correlation, beyond the boundary line meaning the presence of serial correlation. All the consequences demonstrate that there exist long range correlations between volatility processes which validate the clustering and
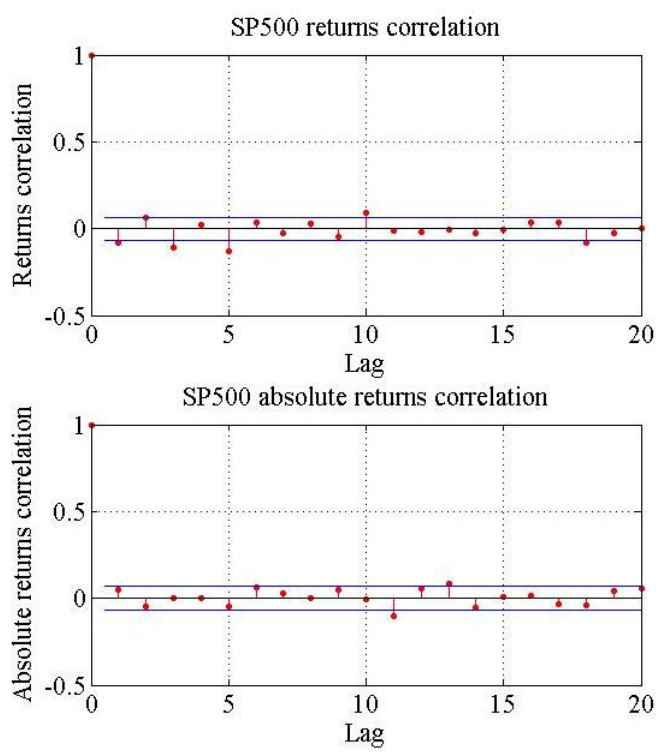

persistence of volatility. While specifically, the long range correlation of SP500 returns process is stronger than the absolute returns process, the similarity of statistical properties of CTS marginal model to returns sequence is better than the similarity of statistical properties of NIG marginal model to the absolute returns series. CTSGOU model identifies correlation of SP500 returns at the lag order 3, 5 and 10, while poor recognition of lag phases for NIGGOU model from absolute returns; therefore it is better to use CTSGOU model for return series model fitting.
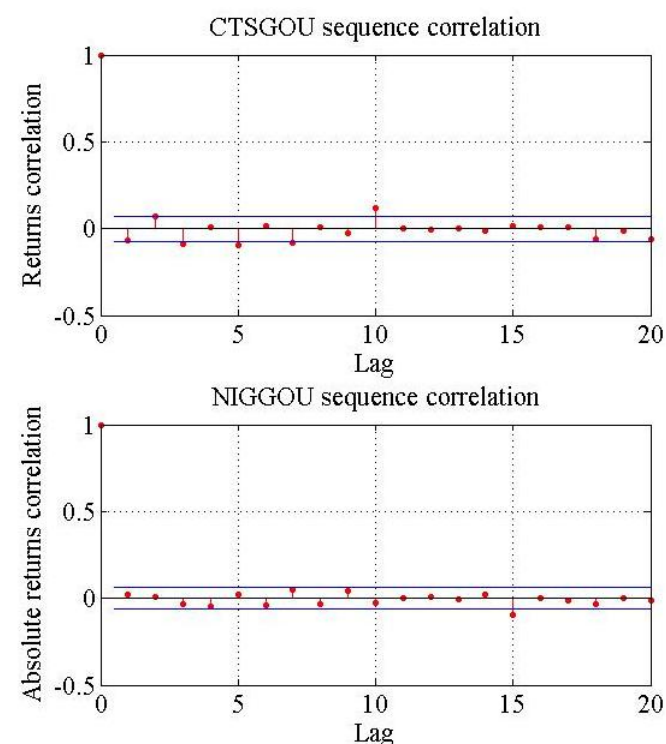

Fig. 3. Correlation functions of returns and absolute returns

\subsection{Option pricing by intelligence optimization}

On numerical experiment we compare the outcomes of calibration procedures. We randomly select market quoted prices of the HSI options traded on Friday 16th October 2015 from Wind database, on which day options with maturities longer than one hundreds days are ruled out for their low volumes. We follow the usual practice assuming 252 trading days with index value 23067.37 that day. The options we use have three expiries: 14 days, 45 days and 76 days, each including 25 different strikes. We run the calibration procedures that contain implementations of PSO algorithm and DE algorithm exploiting characteristic function based FFT pricing method applied to three different models.

The goal of this section is to look for the optimal parameters set that will be used for option pricing in Chinese emerging financial market, we minimize the root mean square error (RMSE) given by formula (20). Alternatively, it can utilize the average relative percentage error (ARPE) denoted as (21). Calibrating an option pricing model means searching parameters set $\Theta$ such that the model's prices are consistent with their market prices, which will result in an optimization problem of the objective function. Because the minimization of equation (20) with respect to the parameters vector $\Theta$ doesn't have an analytical solution, an intelligent optimization routine is needed to find the global minimum. As already observed by Fang et al. [40], this is a well-known ill-posed problem, mainly because the solution is not necessarily unique and there is no guarantee to existence. The objective function is given by RMSE between the market observables and the model prices, and is carried out over all strikes and maturities. To calibrate the parameter set of three LVGOU models with intelligence techniques on dataset, we start with randomly chosen initial parameter sets to search for global minima, and list the calibration results of two simulated methods on three models.

$$
\begin{aligned}
& \operatorname{RMSE}(\Theta)=\sqrt{\sum_{T_{i}} \sum_{K_{j}} \frac{\left(P_{\text {vol }}^{\text {mark }}-P_{\text {vol }}^{\text {model }}\right)^{2}}{N_{\text {obs }}},} \\
& \operatorname{ARPE}(\Theta)=\frac{1}{N_{o b s}} \sum_{T_{i}} \sum_{K_{j}} \frac{\left|P_{\text {vol }}^{\text {mark }}-P_{\text {vol }}^{\text {model }}\right|}{P_{\text {vol }}^{\text {mark }}},
\end{aligned}
$$

where $T_{i}$ and $K_{j}$ are different maturities and strikes, respectively. $N_{o b s}$ denotes the number of observations, and $\Theta$ denotes the parameter vector according to a given model. $P^{\text {mark }}$ and $P^{\text {model }}$ are the option prices of real market and option prices derived from models, respectively.

Table 2 reports the calibration results by intelligence optimization algorithms described above. We first analyze the time changed Classical Tempered Stable model, the CTS process has finite variation since the evaluation of $\alpha$ is 0.5268 and 0.7769 , 
respectively. The density decay rate of right tail $\lambda_{+}=28.8383,32.6519$ are larger than the left one $\lambda_{-}=15.5613,8.4495$, implying more right tail dampening and left skew feature in density distribution. The differences in tails parameters of CTSGOU process give rise to negative skewness. The negative skewness is also captured by time changed Normal Inverse Gaussian model that is reflected by negative estimation of $\beta$ for NIGGOU model. The value of shape parameter $\alpha$ in CTSGOU indicates leptokurtic nature and fat tail phenomenon in returns. The excess kurtosis can also arise from frequent jumps induced by other LVGOU processes.

What we can see from Barndorff-Nielsen and Shephard model is that the jump component has significant intensity and small jumps size with a relatively smaller expectation value. This implies that the compound Poisson process attempts to jump as an infinite activity process that has a large number of small jumps. The diffusion volatility parameter $\xi$ is a little bit large because the diffusion component embraces part of small jumps that should have been captured by jumps component. The correlation coefficient $\rho<0$ confirms the leverage effect existed in return process and variance rate process. The parameter valuation of $\lambda, a$ in infinite models are smaller than those in BNS model, while the long term mean of volatility $1 / b$ is higher than that in BNS. This is because the infinite activity models can capture infinite small jumps and extremely large jumps simultaneously, thus reducing the persistence of the volatility diffusion process.

Among the calibrated GOU based stochastic volatility Lévy processes models, the CTSGOU model exhibits the best calibrating results with respect to RMSE on dataset. The average RMSE pricing error for CTSGOU is 0.2574 , while they are 2.9361 and 7.0410 for NIGGOU and BNS, respectively. The empirical study indicates that infinite activity LVGOU models are preferable to jump-diffusion BNS model in capturing the leptokurtic feature and jumps behaviors despite the presence of diffusion components. We can see that both the two algorithms perform well since they can explain the observed leptokurtic feature of returns and Differential Evolution algorithm performs better with respect to pricing error in empirical researches.

Table 2

Model calibration results

\begin{tabular}{|c|c|c|c|c|}
\hline Model & PSO estimated results & DE estimated results & PSO RMSE & DE RMSE \\
\hline $\begin{array}{c}\mathrm{BNS} \\
(\xi, \rho, \lambda, a, b)\end{array}$ & $\begin{array}{c}(0.3291,-0.5962,6.1524,3.2417 \\
3.7748)\end{array}$ & $\begin{array}{c}(0.4067,-0.3216,7.2493,4.5672, \\
2.3536)\end{array}$ & 7.9571 & 6.1248 \\
\hline $\begin{array}{c}\text { NIGGOU } \\
(\alpha, \beta, \delta, \lambda, a, b,)\end{array}$ & $\begin{array}{c}(4.6103,-0.8249,2.1174,5.3588 \\
0.7192,1.2238)\end{array}$ & $\begin{array}{c}(6.1503,-1.9733,0.8521,3.1026 \\
0.4671,0.8823)\end{array}$ & 4.1501 & 1.7220 \\
\hline $\begin{array}{c}\text { CTSGOU } \\
\left(C, \lambda_{+}, \lambda_{-}, \alpha, \lambda, a, b\right)\end{array}$ & $\begin{array}{c}(2.4892,28.8383,15.5613,0.5268 \\
2.5116,1.0839,3.4710)\end{array}$ & $\begin{array}{c}(3.5217,32.6519,8.4495,0.7769 \\
3.5680,0.8531,2.0846)\end{array}$ & 0.3444 & 0.1703 \\
\hline
\end{tabular}
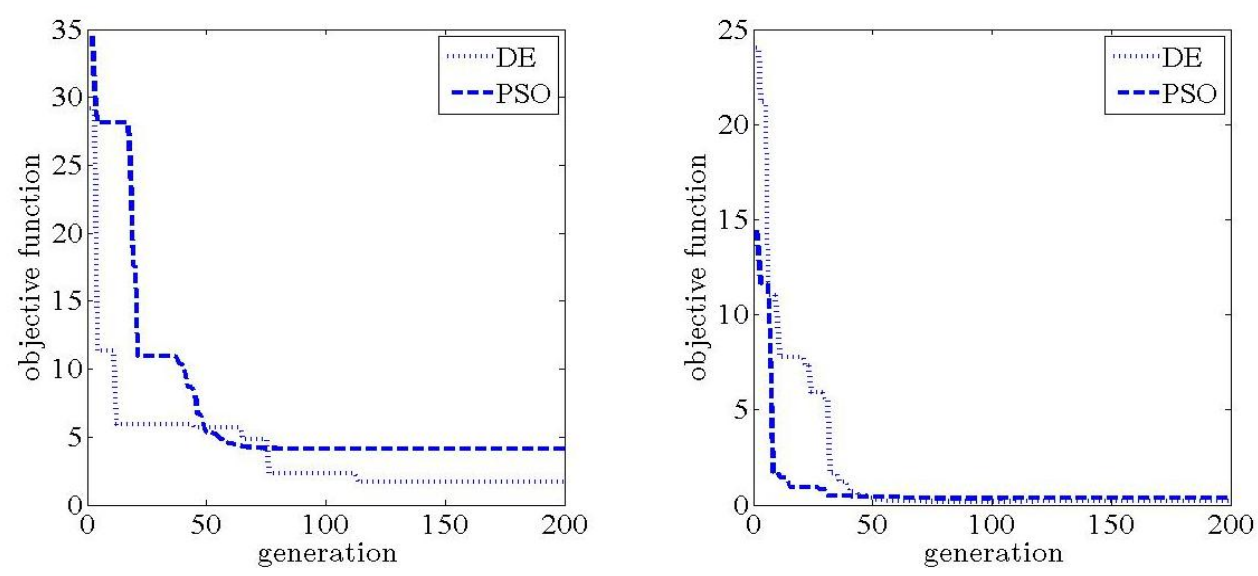

Fig. 4. Convergence of DE and PSO applied to NIGGOU (left panel) and CTSGOU (right panel

In the DE calibration procedure, the specific definition of the crossover probability is 0.8 , the scaling factor $F=0.85$ and the maximum number of iterations is 200. The population size of PSO particles is 50, with also 200 generations. Fig. 4 displays the convergence of intermediate objective function applied to NIGGOU model and CTSGOU model, using PSO and DE algorithms respectively. We can see that it takes roughly 120 generations for CTSGOU model to converge and 110 generations for NIGGOU in $\mathrm{DE}$ to reach a stable minimum value. It takes about 100 generations in both models by PSO for convergence. We first use intelligence global search methods to identify the general location of the global optimum, then use local search algorithm to pinpoint their exact location. The use of PSO is in combination with interior point algorithm, and the use of DE is in combination with Levenberg Marquardt algorithm. It is the hybrid DE algorithm that clearly produces potentially better results. The convergence 
performance of the PSO algorithm is relatively worse for the convergence prematurity. We can conclude that $\mathrm{DE}$ algorithm is more effective than PSO algorithm on this issue.

To further visualize the efficiency of calibration results of intelligence optimization methods, Fig. 5 and Fig. 6 display the calibrated call option prices and implied volatilities of the two simulated methods applied to CTSGOU model, which is superior to other infinite activity jump models and finite jump model at capturing leptokurtosis. It is experimented on the whole ranges of strikes and maturities and has proven that DE technique exhibits superiority over PSO approach. The consequences of other two models are

PSO based Call price surface under CTSGOU model

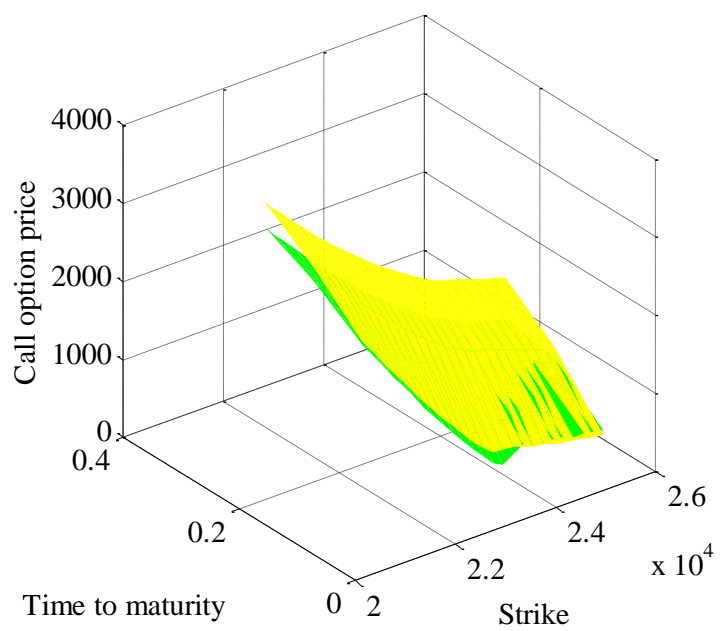

omitted for brevity. We find that both these methods perform relatively better in pricing out of money options and relatively worse in deep in the money options. As is shown in the call option prices figures, the results obtained in Differential Evolution algorithm match closely to the real option prices. It can figure out that Differential Evolution algorithm fits real phenomenon of options closer than Particle Swarm optimization algorithm fitting results except on some noisy implied volatilities on short maturity, and more accurately reflects the volatility smile property. Putting all together, the DE optimization algorithm is preferable to the PSO optimization algorithm that needs to be improved.

PSO based implied volatility surface under CTSGOU model

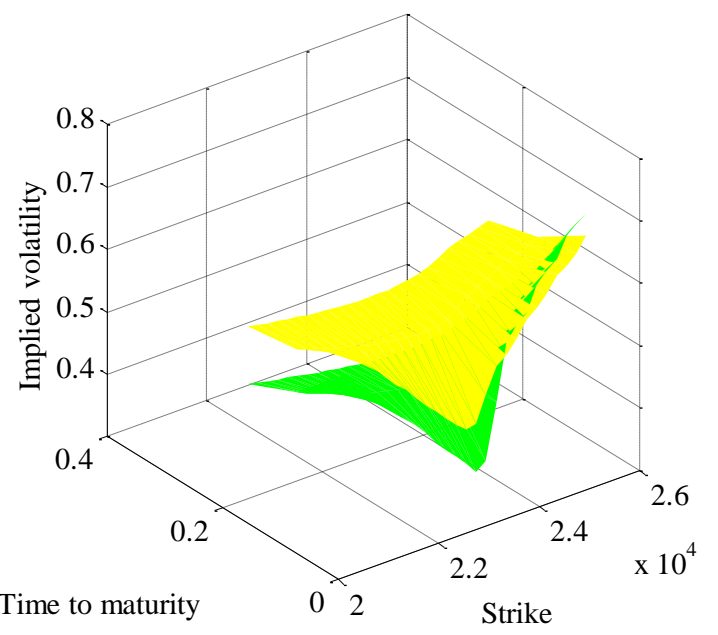

Fig.5. The calibration results of option prices and implied volatilities of PSO approach under CTSGOU model. Green color stands for real option data and implied volatilities of HSI index option, yellow color stands for PSO simulated option prices and implied volatilities.

DE based Call price surface under CTSGOU model

DE based implied volatility surface under CTSGOU model
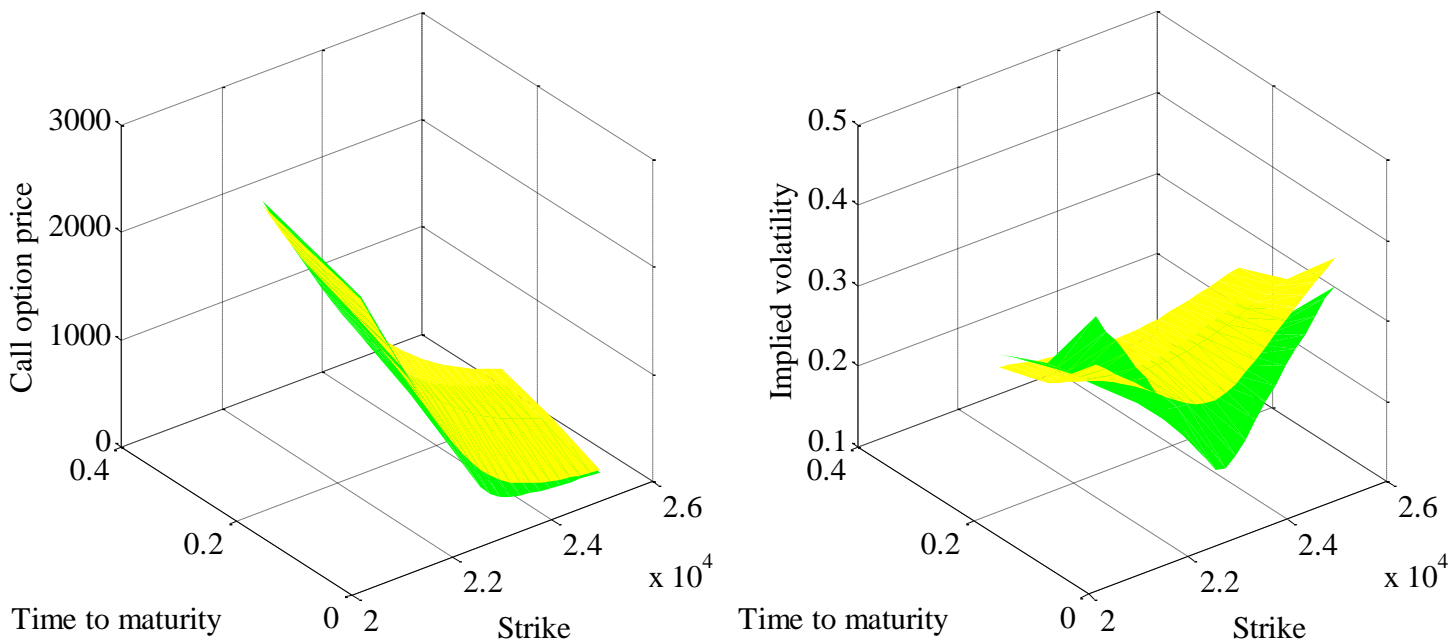

Fig.6. The calibration results of option prices and implied volatilities of DE approach under CTSGOU model. Green color stands for real option data and implied volatilities of HSI index option, yellow color stands for DE simulated option prices and implied volatilities.

\subsection{Option hedging by optimization techniques}

The Lévy driven stochastic volatility models usually correspond to incomplete markets, so that exact replication is unavailable. Therefore, hedging can be explained as approximation of the ultimate payoff with a feasible portfolio. One approach called variance optimal hedging is to take into account a trading strategy that adjusts the global variance to be minimized. Following Kallsen and Pauwels [41], a 
new criterion about the strategy is introduced through minimizing the squared hedging error under risk neutral measure. We need to search a deal strategy $\zeta_{t}$ in collaboration with an initial endowment $V_{0}$ such that

$$
l_{0}=\inf _{\varsigma \in D} \tilde{E}\left[e^{-r T}\left(S_{T}-K\right)^{+}-V_{0}-\int_{0}^{T} \varsigma_{t} d \hat{S}_{t}\right]^{2},
$$

where $D$ is the domain of feasible self financing strategies. The representation in bracket denotes the overall loss of a self financing portfolio, and the solution $\zeta_{t}$ to (22) is the variance optimal strategy. We implement formula (22) in a discrete form that is given by:

$$
\begin{aligned}
\tilde{i_{0}}= & \sum_{t=0}^{\frac{T}{\Delta_{t}}}\left[V_{0}+\varsigma_{t \Delta}\left(S_{t \Delta_{t}} e^{-r t \Delta_{t}}-S_{t\left(\Delta_{t}-1\right)} e^{-r t\left(\Delta_{t}-1\right)}\right),\right. \\
& \left.-e^{-r T}\left(S_{T}-K\right)^{+}\right]
\end{aligned}
$$

where we assume that the hedge position is adjusted every $\Delta_{t}$ days.

To further assess the hedging power of the proposed models, we use the parameters obtained previously and the short maturity option with 25 strikes as well as historical stock prices time series during 20th October 2015 to 20th November 2015 to get the RMSE errors. The volatility used to compute Delta hedging strategy is assumed to be backed out from short maturity options on 20th October 2015. The hedging errors of Delta hedge and variance optimal hedge classified by static hedge, two days intervals and one week intervals are outlined in Table 3.

The evaluations in each line of Table 3 correspond to the same hedging strategy through different adjustment interval. We can see that the values decrease with the adjustment frequency increasing, meaning reduced hedge cost for traders. The infinite activity Lévy driven stochastic volatility models perform better than the BNS model in long intervals rebalanced hedge, whereas showing relatively poorer results in frequent rebalanced hedge. What can be inferred from this is that BNS model fits more appropriately for short maturity options than NIGGOU and CTSGOU model. During the optimal hedge examination, CTSGOU model has the least average error in contrast to others. Additionally, the inclusion of infinite Lévy jumps does enhance the ability to manage market risks.

\begin{tabular}{|c|c|c|c|}
\hline \multirow[t]{2}{*}{ Strategies } & \multicolumn{3}{|c|}{ RMSE hedging errors } \\
\hline & Static Hedge & One week intervals & Two days intervals \\
\hline CTSGOU Optimal & 0.0319 & 0.0307 & 0.0285 \\
\hline NIGGOU Optimal & 0.0538 & 0.0432 & 0.0366 \\
\hline BNS Optimal & 0.0651 & 0.0504 & 0.0217 \\
\hline Delta Hedge & 0.0825 & 0.0745 & 0.0638 \\
\hline
\end{tabular}

Table 3

RMSE hedging errors in intervals categories

\subsection{Out-of-sample option pricing performance}

We conduct out-of-sample forecast to examine the efficiency of the empirical consequences obtained above so as to avoid over-fitting misspecifications that may also achieve good results by chance. The experiment is made on the next day, namely Monday 19th October 2015, with short term maturity and medium term maturity options considered similarly. The HSI on that day is 23075.61. For comparative purpose, we exhibit the pricing errors values as measures for wide ranges of models.

We investigate ARPE and RMSE produced from the calibrated parameters for the next trading day. The out of sample pricing errors with 44 days expiries and 75 days expiries are respectively presented in Table 4 by maturity categories and moneyness (K/S) classifications. The calculation results reported in Table 4 show infinite activity models give smaller error in DE technique than Black-Sholes (BS) and BNS models overall. The respective average RMSE errors for CTSGOU and NIGGOU are 3.2615 and 3.4408, whereas 4.3909 and 6.8868 for BS and BNS models. Specifically, infinite jumps models give significant smaller pricing errors for short maturity and medium maturity options compared to BS and BNS models for ATM and OTM options. It is illustrated that stochastic volatility models in combination with infinite activity Lévy jumps add explanatory power to improve empirical fits efficiency.

Nevertheless, the pricing errors of all the models become smaller for ITM options. The average ARPE value of BS model is $44.35 \%$ and $27.44 \%$ smaller than that of NIGGOU and CTSGOU models respectively for ITM options, whereas for OTM options, the average ARPE values of NIGGOU and CTSGOU models is $37.74 \%$ and $47.61 \%$ smaller than that of BS model, indicating that infinite activity Lévy process display preponderance in OTM option forecast. The same conclusion can be drawn in RMSE calculation. The average RMSE value of infinite Lévy models with medium maturity are $2.02 \%$ larger than the corresponding values for short maturity options. Hence, LVGOU models are more suitable for short expiries options pricing. We can confirm that the infinite activity LVGOU exhibits good performers across strikes and maturities, producing smaller errors 
than other models by contrast to market observations.

Table 4

Pricing errors in moneyness and maturity categories

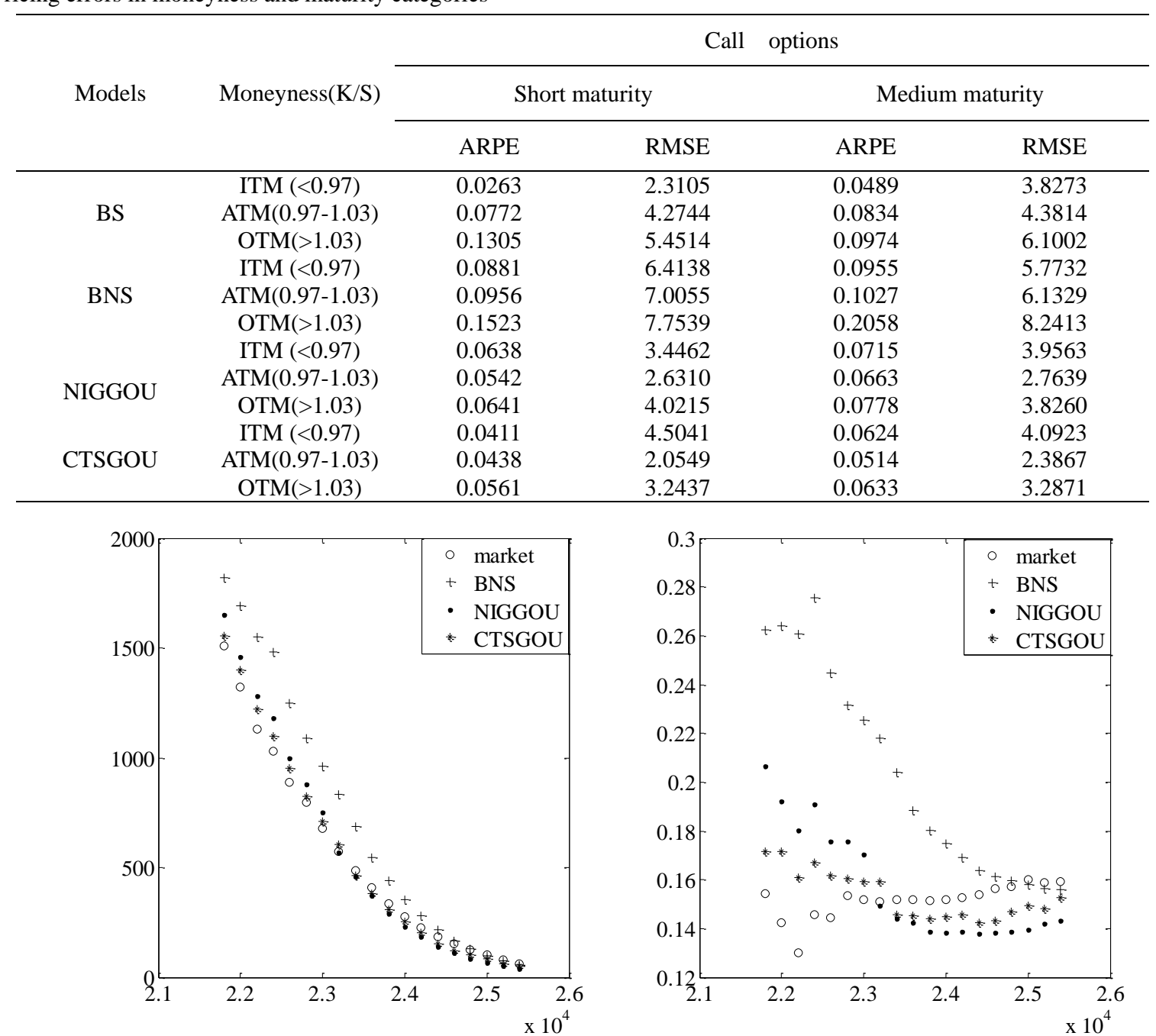

Fig. 7. Out-of-sample option pricing results and implied volatility performance

Fig. 7 plots the HSI out-of-sample option pricing performance and implied volatility structures for short maturity options using three time changed Lévy models. It can be observed that the model produced option prices in CTS marginal model fit market options best, and are significantly consistent with market prices at the money. Therefore, it demonstrates that the CTSGOU model generates more realistic behaviors as well as better fit to observations.

\section{Conclusion}

The fact that normal innovations in BS option pricing model cannot incorporate volatility smile and excess kurtosis has led to various alternative models. We combine OU based stochastic volatility models and infinite activity Lévy processes in constructing time changed Lévy models models including GOU based Normal Inverse Gaussian model and GOU based Classical Tempered Stable model. Additionally, Barndorff-Nielsen and Shephard model is added to compare this wide range of models for option pricing and hedging using returns data and options data.
With the heuristic optimization methods comprised of hybrid Particle Swarm optimization algorithm and hybrid Differential Evolution algorithm where both complementary strengths of approaches can be utilized from quick search, experiments have been made to the three proposed LVGOU models through characteristic functions based FFT calculation. The experiments come out that hybrid DE algorithm works relatively better to LVGOU models. Comparison of the performance of three Lévy driven stochastic volatility processes reveals that the CTSGOU model has the smallest pricing deviations to observables.

\section{Acknowledgments}

We would like to acknowledge the financial support from the National Science Foundation of China (No. 71171042, 71271047, 71571038).

\section{References}

[1] Carr P, Geman H, Madan DB, Yor M. The fine structure of asset returns: An empirical investigation. J Bus 2002; 75: 
205-332.

[2] Sahalia YA, Jacod J. Estimating the degree of activity of jumps in high frequency data. Ann Stat 2009; 37: 2202-2244.

[3] Klingler S, Kim YS, Svetlozar T. Option pricing with time-changed Lévy processes. App Financ Econ 2013; 23: $1231-1238$.

[4] Kou SG, Wang H. Option pricing under a double exponential jump diffusion model. Manag Sci 2004; 50: $1178-1192$.

[5] Mozumder S, Sorwar G, Dowd K. Option pricing under non-normality: a comparative analysis. Rev Quan Finance Accout 2013; 40: 273-292.

[6] Shi QH, Yang XP. Pricing Asian options in a stochastic volatility model with jumps. Appl Math Comput 2014; 228: 411-422.

[7] Abdelrazeq I. Model verification for Lévy-driven Ornstein-Uhlenbeck processes with estimated parameters. Statist Prob Lett 2015; 104: 26-35.

[8] Carr P, Wu L. Time-changed Lévy processes and option pricing. J Finan Econ 2004; 71: 113-141.

[9] Valenti D, Spagnolo B, Bonanno G. Hitting time distributions in financial markets. Physica A 2007; 382: 311-320.

[10] Bonanno G, Valenti D, Spagnolo B. Role of noise in a market model with stochastic volatility. Eur Phys J B 2006; 53: 405-409.

[11] Spagnolo B, Valenti D. Volatility effects on the escape time in financial market models. Int $\mathbf{J}$ Bifurcation Chaos 2008; 18: 2775-2786.

[12] Bonanno G, Valenti D, Spagnolo B. Mean escape time in a system with stochastic volatility. Phys Rev E 2007; 75: 016106 (8).

[13] Schoutens W, Symens S. The pricing of exotic options by Monte-Carlo simulations in a Levy market with stochastic volatility. Int J Theo Appl Finance 2003; 6: 839-864.

[14] Bianchi ML, Fabozzi FJ. Investigating the performance of non-Gaussian stochastic intensity models in the calibration of credit default swap spreads. Comput Econ 2015; 46: 243-273.

[15] Barndorff-Nielsen OE, Nicolato E, Shephard N. Some recent developments in stochastic volatility modeling. Quan Finan 2002; 2: 11-23.

[16] Creal DD. Analysis of filtering and smoothing algorithms for Lévy-driven stochastic volatility models. Comput Stat Data Anal 2008; 52: 2863-2876.

[17] Todorov V. Econometric analysis of jump driven stochastic volatility models. J Econometrics 2011; 160: 12-21.

[18] Andreas KA, Carol. Stochastic volatility jump-diffusions for European equity index dynamics. Euro Finan Manag 2013; 19: 470-496.

[19] Huang JX, Zhu WL, Ruan XF. Option pricing using the fast Fourier transform under the double exponential jump model with stochastic volatility and stochastic intensity. J Comput Appl Math 2014; 263: 152-159.

[20] Barndorff-Nielsen OE. Processes of normal inverse Gaussian type. Finance Stoch 1997; 2: 41-68.

[21] Rosinski J. Tempering stable processes. Stoch Procr Appl 2007; 117: 677-707.

[22] Dubkov AA, Spagnolo B, Uchaikin VV. Lévy flights superdiffusion: an introduction. Int $\mathrm{J}$ Bifurcation Chaos 2008; 18: 2649-2672.

[23] Kim YS, Rachev S, Bianchi M. Time series analysis for financial market meltdowns. J Banking Finan 2011; 35: 1879-1891.

[24] Kuchler U, Tappe S. Tempered stable distributions and processes. Stoch Proc Appl 2013; 123: 4256-4293.

[25] Zaevski TS, Kim YS, Fabozzi FJ. Option pricing under stochastic volatility and tempered stable Lévy jumps. Int Rev Finan Anal 2014; 31: 101-108.

[26] Li J, Faverob C, Ortu F. A spectral estimation of tempered stable stochastic volatility models and option pricing. Comput Stat Data Anal 2012; 56: 3645-3658.

[27] Liang CX, Li SH. Option pricing and hedging in incomplete market driven by Normal Tempered Stable process with stochastic volatility. J Mathe Anal Appl 2015; 423: 701-719.

[28] Yu CL, Li HT, Wells MT. MCMC estimation of Levy jump models using stock and option prices. Math Finance 2011; 21: 383-422.

[29] Taufer E, Leonenko N. Simulation of Lévy-driven Ornstein-Uhlenbeck processes with given marginal distribution. Comput Stat Data Anal 2009; 53: 2427-2437.

[30] Kawai R, Masuda H. Infinite variation tempered stable Ornstein-Uhlenbeck processes with discrete observations. Commu Stat Simu Comput 2012; 41: 125-139.

[31] Zhang WG, Liu YJ, Xu WJ. A new fuzzy programming approach for multi-period portfolio optimization with return demand and risk control. Fuzzy Set Syst 2014; 246: 107-126.

[32] Yang S, Lee J. Multi-basin particle swarm intelligence method for optimal calibration of parametric Levy models. Expert Sys Appl 2012; 39: 482-493.

[33] Fastrich B, Winker P. Combining forecasts with missing data: making use of portfolio theory. Comput Econ 2014; 44: $127-152$.

[34] Sato K. Lévy Processes and Infinitely Divisible Distributions. Cambridge: Cambridge University Press; 1999.

[35] Panda S, Padhy NP. Comparison of particle swarm optimization and genetic algorithm for FACTS-based controller design. Appl Soft Comput J 2008; 8: $1418-1427$.

[36] Price K, Storn R, Lampinen J. Differential evolution: A practical guide to global optimization. Berlin: Springer; 2005.

[37] Krink T, Paterlini S. Multiobjective optimization using differential evolution for real-world portfolio optimization. Comput Manag Sci 2011; 8: 157-179.

[38] Carr P, Madan D. Option valuation using the fast Fourier transform. J Comput Finance 2001; 2: 61-73.

[39] Lord R, Kahl C. Optimal Fourier inversion in semi-analytical option pricing. J Comput Finance 2007; 10 : $1-30$.

[40] Fang F, Jonsson H, Oosterlee K, Schoutens W. Fast valuation and calibration of credit default swaps under Levy dynamics. J Comput Finance 2010; 14: 57-86.

[41] Kallsen J, Pauwels A. Variance-optimal hedging for time-changed Lévy processes. Appl Math Finance 2011; 18: $1-28$. 